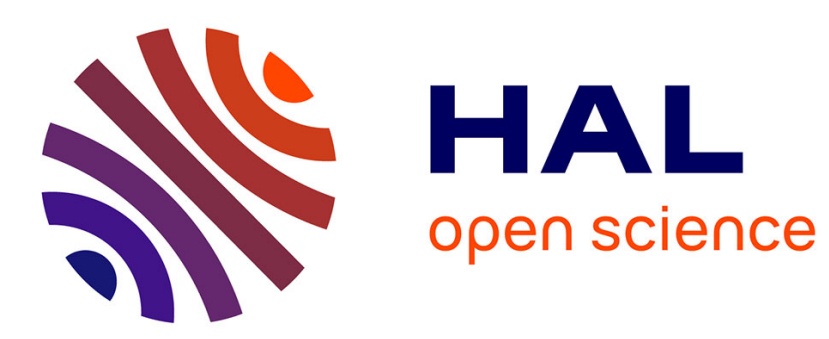

\title{
Le féminin maternel chez les femmes toxicomanes sous substitution
}

Ouriel Rosenblum

\section{To cite this version:}

Ouriel Rosenblum. Le féminin maternel chez les femmes toxicomanes sous substitution : De la passion du corps à la resexualisation de la relation. Dialogue , 2009, Peur de nos enfants ?, 2 (184), pp.115-129. 10.3917/dia.184.0115 . hal-01517004

\section{HAL Id: hal-01517004 https://hal.science/hal-01517004}

Submitted on 26 Jun 2017

HAL is a multi-disciplinary open access archive for the deposit and dissemination of scientific research documents, whether they are published or not. The documents may come from teaching and research institutions in France or abroad, or from public or private research centers.
L'archive ouverte pluridisciplinaire HAL, est destinée au dépôt et à la diffusion de documents scientifiques de niveau recherche, publiés ou non, émanant des établissements d'enseignement et de recherche français ou étrangers, des laboratoires publics ou privés. 


\section{Le féminin maternel chez les femmes toxicomanes sous substitution De la passion du corps à la resexualisation de la relation}

OURIEL ROSENBLUM

La toxicomanie peut être une figure paradigmatique de la stratégie que met en place une femme, en proie à l'effraction du sexuel génital, pour aborder les rivages du féminin maternel. Nous tenterons, à partir d'une analyse de la trajectoire des femmes toxicomanes, de la consommation à la substitution en passant par la maternité, d'aborder la narrativité de soi comme une resexualisation du corps et de la pensée.

\section{La toxicomanie au féminin : un pont entre la sexualité et le désir d'enfant}

Si les conduites addictives établissent une stratégie pour retarder l'entrée dans le champ de la sexualité adulte, la rencontre avec l'héroïne signe une satisfaction des besoins qui vient faire l'impasse sur le désir engagé par le corps sexué.

Rencontre avec l'héroïne objet de la jouissance, anatomie du plaisir sans désir ni sexuation

Il existe un lien entre les conduites addictives et celles agies autour du corps telles que l'anorexie ou la boulimie; la désexualisation du corps sculpte une 
identité sexuée ambiguë qui, chez la femme toxicomane, participe à une non-détermination, à un aspect androgyne de la silhouette. Ainsi, la consommation d'héroïne pourrait venir occulter la question laissée sans réponse de la nature de la différence entre les sexes.

\section{Sexualité et toxicomanie au féminin}

Selon P. Angel (2000), la sexualité des femmes toxicomanes est caractérisée par une diminution de la libido, l'investissement étant déplacé vers le produit. L'activité sexuelle s'exercerait parfois sur un mode compulsif, les partenaires sont alors multiples, interchangeables, peu ou non reconnus dans leur individualité.

O. Thomas (2006) souligne, en outre, l'évocation fréquente de récits de traumatismes sexuels subis durant l'enfance ou à la période de l'adolescence. On pourrait alors émettre deux séries d'hypothèses. La première serait que la prise de drogue aurait pour fonction de venir apaiser la douleur psychique provoquée par le traumatisme. La deuxième s'appuierait sur la fonction d'oubli du souvenir du traumatisme. Mais, selon cet auteur, la logique passionnelle de la toxicomanie féminine se révèle dans le travail psychothérapeutique sous le couvert du traumatisme, dans sa fonction de voile et dans son expression dans l'amour et la haine de transfert.

Le récit rapporté par les femmes toxicomanes des rapports sexuels est souvent infiltré de thématiques associant le viol et la frigidité, dont l'illustration est retrouvée de manière caricaturale dans les fréquentes activités de prostitution où le corps sexué est déconsidéré, sans valeur ni féminité. De plus, l'absence ou l'irrégularité des règles en raison de la consommation d'hérö̈ne vient confirmer à la fois le déni de la féminité et la certitude de ne pouvoir engendrer. L'illusion d'invulnérabilité, de complétude, apportée par l'usage du produit, l'aménorrhée qui découle de cette consommation, concourent à l'illusion d'une stérilité. L'aspect androgyne, les conduites anorexiques, la dégradation physique et les attaques contre le corps (injections, infections...) traduisent les difficultés d'identité de la plupart de ces femmes. Souvent ces dernières ont des antécédents d'IVG. Les grossesses non abouties à répétition, dans un contexte de délabrement du corps, peuvent leur permettre une vérification de l'intégrité des fonctions reproductives sans réel investissement de celles-ci.

\section{Le destin du couple au sein du parcours toxicomaniaque}

Selon N. Losito et L. Gibier (1999), la notion de trajectoire du toxicomane est utile dans la compréhension de la formation et de la structuration d'un couple. En effet, un couple qui se constitue lors de la phase de «lune de miel » n'aura pas les mêmes assises qu'un couple qui se forme au moment 
de la période de "galère »; dans le premier cas de figure, l'objet drogue peut faire partie du mythe fondateur : «C'est mon ami qui m'a fait mon premier shoot. » La plupart des études insistent sur la rareté des relations sexuelles, l'acquisition et la consommation du toxique régissent les rapports à l'intérieur de ces couples.

\section{La place du désir d'enfant au cours de l'addiction}

Dans ce contexte, l'enfant peut être investi comme «pare-drogue » en place et lieu du produit, il sera vécu comme sauveteur. Chez la femme toxicomane, le désir d'enfant, de même que la grossesse-accident, peut signifier qu'elle tente de trouver à l'extérieur d'elle la butée qu'elle ne trouve pas en son for intérieur pour arrêter la consommation. Parfois, la compétition entre le désir d'enfant et la dépendance au toxique se traduit par l'alternance entre démarches de sevrage et épisodes d'intoxication massive.

L'enfant est investi d'une fonction de réparation qui va défendre la femme contre l'agressivité et les sentiments de culpabilité qu'elle a développés à l'encontre de sa propre mère, et vient modérer l'appétence toxique en comblant les désirs de plénitude recherchée. Pour une femme toxicomane, un des enjeux du désir d'enfant serait alors une possibilité d'engager des retrouvailles avec l'objet perdu, la dépendance au produit venant masquer la difficulté à élaborer la perte. En effet, si pour toute femme la grossesse est le moment privilégié d'un rapproché avec l'image de sa propre mère, pour une toxicomane cet enjeu est d'autant plus présent que la relation à la mère demeure assez fusionnelle. Ainsi, la stratégie mégalomaniaque du toxicomane avec la toute-puissance conférée au produit vient dénier la dépendance des premières relations mère-enfant.

\section{Rôle du produit de substitution dans l'accès à la parentalité}

À partir de l'analyse du discours autobiographique des femmes toxicomanes, nous avons constaté que celles-ci différencient clairement la période d'intoxication active de celle de la substitution (O. Rosenblum, 2004). Au cours du temps de la toxicomanie, elles ont mis entre parenthèses leur féminité, elles effectuent une association entre leur féminité déniée et une maternité inabordable. Elles ont en outre une perception manichéenne de l'enfant, percevant avec difficulté ses besoins propres et le considérant comme un futur sauveur. Elles établissent un lien entre le désir d'enfant et le besoin de dépendance. Elles éprouvent un sentiment de culpabilité vis-à-vis d'un futur enfant, lié à leur consommation. En revanche, l'accès à une substitution leur permet à la fois une construction identitaire avec une découverte de leur image corporelle renforçant leur estime de soi - les positionnant au sein d'une place active 
féminine - et l'accession à une parentalité plus affirmée avec une intériorisation des différentes étapes du devenir parent et un réordonnancement de la chaîne des générations où les grossesses sont investies sous le sceau de la substitution.

Ainsi, le produit de substitution (méthadone ou Subutex) permet l'introduction d'une fonction de parrainage pour les futurs parents auprès de l'enfant au sein d'un engagement dans une filière de soins spécifiques. Cette substance médicamenteuse proche du toxique, labélisant le statut du toxicomane engagé dans la procréation, autorise la poursuite d'un processus de réaffiliation incarné par le devenir parent chez des individus s'estimant constamment en danger de désaffiliation.

Travaillant sur la nature de la spécificité de l'accès au devenir parent des femmes toxicomanes, nous avons remarqué que le récit de la trajectoire des mères rencontrées depuis l'entrée dans la toxicomanie jusqu'à la mise en place de la substitution comportait une richesse de nature à rendre intelligible les aléas de l'accès à la parentalité. Une mise en perspective de la totalité de leur trajectoire nous est apparue alors plus féconde qu'une focalisation sur le seul moment du traitement à la méthadone.

\section{Le récit de la trajectoire comme mise en narration de l'identité sexuée}

En collaboration avec l'École des hautes études en sciences sociales, le Centre Pierre Nicole à Paris et le laboratoire PSITEC à Lille 3, nous avons effectué un travail de recherche autour du parcours d'une population de quinze femmes toxicomanes, confrontées à leur parentalité à un des moments-clés de leur existence : celui où elles ont décidé d'interrompre leur consommation active. Pour y parvenir, elles fréquentent un centre spécialisé leur proposant un traitement de substitution.

Nous avons procédé à l'analyse du discours autobiographique des femmes, interrogées à la fois sur leur période de consommation de drogues et sur celle contemporaine de la substitution. Nous avons cherché à découvrir d'une part si, malgré la diversité de leurs trajectoires, on ne retrouverait pas des événements de vie susceptibles d'être regroupés, en rapport à l'accès à leur parentalité, par la voie du maternel féminin. Nous avons étudié d'autre part en quoi l'accès à la méthadone, produit de substitution employé dans ce centre, affecte ou non leur rapport à la maternité, à la représentation de leur féminité et, d'une manière plus générale, à leur environnement. Enfin, nous avons tenté d'apprécier également les spécificités propres à ces femmes qui évoluent dans le temps, tel le rapport au corps féminin, à la procréation, aux enfants, à l'autre et aux institutions, en prenant comme paramètre principal l'accès à la substitution. 
Par cette approche qualitative, nous avons pu faire émerger quelques facteurs de changement au sein d'une trajectoire spécifique : la femme toxicomane ayant accès au féminin maternel. Le parcours de la toxicomanie décrit ici peut représenter l'une des figures paradigmatiques illustrant les aléas de l'accession à la parentalité et les risques de désaffiliation. Il ne s'agit pas d'aborder les pratiques, mais plutôt de saisir la dimension expérientielle et de parvenir aux représentations attachées à cette période de la vie. Les entretiens semi-directifs centrés sur l'accès à la parentalité étant une des voies d'accès privilégiée afin d'aborder l'histoire et le ressenti de ces femmes. Les principaux thèmes concernaient les représentations que la femme toxicomane peut avoir dans ses rapports à la fois à son corps, aux atteintes somatiques, à son identité féminine, à l'enfant, à l'homme, à sa famille, aux institutions, aux soins, à la drogue...

Par l'étude de la trajectoire des mères toxicomanes, nous avons appréhendé la diversité de leurs parcours. Nous avons abordé et développé les thèmes qui nous semblent les plus caractéristiques des aléas auxquels sont confrontées ces femmes tout au long de ce parcours, tels que les difficultés de l'investissement du corps féminin, un accès à la procréation qui s'élabore difficilement, une parentalité malmenée, des relations problématiques aux proches. À partir de ces thèmes, nous avons tenté de décrire des configurations au sein des trajectoires qui peuvent qualifier les différents modes d'accès à la parentalité de ces mères. En effet, toutes les mères rencontrées ont un accès identique au traitement de substitution par la méthadone. C'est une situation à la fois normative : une mère toxicomane se soigne et, entraînant une stigmatisation, l'accès au traitement de substitution indique l'état toxicomaniaque du sujet. Dans ce contexte, nous avons cherché à discerner, à travers le discours des mères, l'impact de la situation de substitution sur l'accès à leur féminité, à leur maternité, à leur parentalité, au mode de relation avec leur conjoint, leurs enfants, leur entourage et l'institution.

\section{Analyse des trajectoires}

Lorsque l'on observe la trajectoire des femmes rencontrées, nous constatons d'une part que les périodes d'incarcération jalonnent le parcours du toxicomane et que d'autre part le maintien d'une vie de couple joue un rôle protecteur vis-à-vis de la garde des enfants. En revanche, les atteintes somatiques chroniques et les troubles psychiques nécessitant une hospitalisation sont des facteurs qui ne jouent pas en faveur d'un accès aisé à une parentalité responsable.

Globalement, les changements constatés par ces femmes évoquent une dynamique, une modification qui va dans la direction souhaitée. Mais une lecture plus attentive nous fait apparaître, de façon significative, que certaines situations relationnelles engagées par les mères sont bien davantage modifiées 
que d'autres. C'est le cas, en particulier, du mode relationnel aux enfants, du regard posé sur elles-mêmes et, dans une moindre mesure, de la relation à leur propre mère. Par ailleurs, nous notons que les femmes rencontrées tentent d'intégrer la notion de temps et de durée dans leurs nouveaux modes relationnels. Nous pouvons synthétiser les principales situations relationnelles où les changements sont perçus par nos sujets, vis-à-vis :

- de la relation à leurs enfants, les motifs évoqués concernent la notion de responsabilisation avec l'accès à une culpabilité témoignant d'une prise de conscience douloureuse, la mise en place des limites et de l'autorité, la capacité de se projeter dans l'avenir par étapes progressives ;

- de la relation à soi-même qui est transformée par l'écoute accrue de l'intériorité du corps, de la découverte des sensations corporelles et du plaisir associé. Le corps devient l'objet de soins, avec un respect de son intégrité et les indices corporels de la féminité sont recherchés ;

- de la relation à leur propre mère qui se traduit par un désir de renouer des liens et une volonté d'occuper une place au sein des générations. Dans le cas particulier où les enfants sont à la charge de la grand-mère maternelle, on note une volonté de réintégrer une place de mère auprès des enfants.

Il n'est pas inintéressant de constater, en revanche, que le rapport à la consommation de drogues n'a pas été transformé radicalement par la prise de méthadone. Ainsi, la plupart des femmes rencontrées, tout en bénéficiant de ce traitement, n'ont pas interrompu totalement leur consommation d'opiacés. La formule la plus souvent évoquée ici concerne la " gestion » des produits.

Concernant les motifs d'accès à la substitution invoqués par les sujets, on perçoit que la plupart des mères rencontrées ont pris elles-mêmes l'initiative, très souvent à l'occasion d'une nouvelle grossesse, de prendre contact avec le centre. Ainsi, pour la majorité de ces femmes, le moment choisi, ici l'accès à la maternité, a été prépondérant pour aborder un processus de soins novateur, qui devrait déterminer une dynamique mutative dans laquelle la femme toxicomane va s'engager tout entière.

À partir de l'analyse de ces trajectoires, nous allons tenter, avec une approche socio-anthropologique et prenant appui sur l'enseignement d'I. Théry à l'EHESS, d'aborder la façon dont le "féminin maternel » navigue ici entre les normes sociétales et la resexualisation de la pensée.

\section{La quête de l'institution}

Au cours de l'analyse des trajectoires, nous avons remarqué que ces femmes étaient en recherche d'institution. En effet, elles nous disent qu'elles ont bénéficié de peu de transmission, que quelque chose ne leur a pas été 
transmis. Elles demeurent collées à leur mère en lien avec leur difficulté d'être elles-mêmes mères. Le maintien dans le milieu de la toxicomanie peut signifier qu'il ne leur reste que le monde non socialisé, comme l'indiquent dans leurs œuvres Kafka et Proust. À l'instar de ces auteurs qui pensent la douleur de leur propre vie et pour qui la littérature est une question de vie ou de mort, l'échange institué n'a pas pu avoir lieu pendant l'enfance. Par ce recours à l'institution, ces femmes nous indiquent le travail à travers le processus d'individuation qui passe par les institutions auxquelles les mères ont accès. L'institution, ici, c'est ce qui inscrit les relations intersubjectives en leur pourvoyant une signification, « être sous substitution »; il s'agit bien ici d'une dimension du social.

\section{L'internalisation des normes}

Les femmes rencontrées internalisent sans cesse des normes, des formes nouvelles de contrôle de l'individu (Elias, 1991). En effet, elles participent au mouvement séculaire d'intériorisation des normes avec, ici, une pénalisation des conduites. Elles se mettent en position de l'obligation de soins, alimentant la politique du soupçon. Elles affirment posséder en elles-mêmes la source de la norme avec en sus une dose de psychologisation. Ainsi, elles sont le témoin de l'encadrement social du désir. Ce cadre est garant du fait qu'elles ne cèdent pas à leur corps, elles se mettent ardemment au service du contrôle social. Les femmes rencontrées s'imposent des contraintes, elles témoignent que les normes se situent à l'intérieur de l'individu. Elles adhèrent, par conséquent, à une forme consentie de contrainte.

\section{La recherche du consentement}

$\mathrm{Au}$ départ, pour ces femmes, le consentement est admis dans les formes sociales et non dans l'intériorité des sentiments, alors que leurs mères sont issues de la génération de la troisième révolution du consentement, celle où l'on assiste à la déliaison entre l'institution matrimoniale et le versant autorisé de la sexualité. Les femmes toxicomanes rencontrées signent leur aliénation en nous montrant que si elles désirent elles ne peuvent consentir, puisqu'elles sont dépendantes du produit qui les aliène. Paradoxalement, c'est une nouvelle forme de consentement : ici, le consentement fait le permis, et l'un des signes du consentement au féminin, c'est l'accès à la santé.

\section{La rencontre avec l'institution permet le changement et la mise en mouvement}

Dans une perspective socio-historique du mouvement, la société est un flot continu en mouvement entre ce qui s'est passé et ce qui n'existe pas encore, 
avec une idée d'interdépendance, montrant là qu'il n'existe pas de société immuable. La sortie de la toxicomanie en est un reflet. En effet, on sort de la stabilité et l'immuabilité du monde biologique incarné par l'état toxicomane pour parvenir au changement et à la diversité humaine. La découverte opérée par la femme toxicomane est de l'ordre du changement historique. Avec la méthadone, elle va connaitre un désenchantement de son monde, une laïcisation des rapports, une relation privilégiée avec des professionnels spécialisés. Ainsi, elle sort du déterminisme anhistorique et des invariants induits par les effets des drogues, au-delà du social ; elle quitte le déterminisme causaliste.

Elle entreprend alors un travail sur sa capacité de réflexivité. La sortie de la toxicomanie s'apparente au doute cartésien : « Je ne tiens rien pour acquis. » Il s'agit d'un travail de substantialisation entre l'intériorité et l'extériorité, l'esprit étant séparé. On peut dire ici avec I. Théry (2001) que c'est le changement dans son ensemble qui opère.

\section{L'émancipation du biologique}

Les femmes rencontrées décrivent la période de toxicomanie active comme une biologisation de l'identité, de la reproduction (IVG répétitives) et de la sexualité (prostitution), la création du manque neuropsychologique, une désaffiliation culturelle, un appel à la médecine comme institution, une création de nature "physiologique » de la dichotomie de l'être, pour signifier l'existence ( « en manque ou pas », « en avoir ou pas »). Elles nous indiquent ainsi une vérité du biologique qui, lui, ne ment pas.

À partir de la période de la substitution, elles décrivent une séparation du biologique, où la méthadone passe du statut de substance à celui de parrain, accompagnant le processus de parentalisation. On assiste à la mise en place de rituels d'initiation avec la fabrication du corps par la prise de méthadone. Ainsi, ces personnes se dégagent de la construction arbitraire du biologique qui les astreignait, dans une polarité univoque, à être en manque ou pas, à être stérile ou non. Elles nous indiquent ainsi comment s'extraire du substrat biologique dont est prisonnier leur corps. Jusque-là, le corps était dévalorisé comme indice de leur infériorité biologique. Ainsi, d'une manière contemporaine à l'accès au traitement de substitution, ces mères n'éprouvent plus l'obligation de "fonctionner » à partir d'un donné du biologique. On perçoit ici qu'un véritable changement du rapport au biologique opère. Cette émancipation se traduit par la mise en place de relations différenciées entre les sexes par le biais d'une réappropriation des figures du féminin. 


\section{Recréation de nouvelles configurations de la dichotomie}

Cet accès à la parentalité avec l'appui de la substitution ouvre le seuil qui franchit l'étape de la biologisation des dichotomies décrite plus haut, par le biais du passage par l'institution. Celle-ci faisant césure entre l'avant et l'après-méthadone, elle permet d'accéder au rivage de l'abord relationnel des dichotomies. Ces femmes évoquent électivement la relation aux enfants, à elles-mêmes, aux figures identificatoires, à l'homme...

Ainsi, c'est là une construction de la différence et la politique de soins prônant l'accès à la substitution est bien un système qui fabrique de la différence. On sortirait de la naturalisation des différences pour parvenir à une « repassionnalisation » des dichotomies par l'abord du jeu relationnel. Ces femmes quittent là l'obligation de rejouer dans la réalité les différentes catégories caricaturales de femmes issues des fantasmes telles que la prostituée, la mauvaise ou la bonne mère, la sorcière empoisonneuse, la femme androgyne...

\section{L'appropriation de la réalité de la différence des sexes}

Par le truchement du discours relatant le mode d'accès à la substitution, on observe une désubstantialisation de la question du sexe. Ces femmes s'extraient du continuum du genre, attributs de l'un et l'autre, dont la figure centrale de la perfection est l'androgyne, selon S. Steinberg (2001). Dorénavant, elles acceptent la nature instable des caractéristiques masculin-féminin. En effet, auparavant, le rapport au corps de la femme toxicomane active se situait bien dans un registre où sa féminité, engagée dans la relation à l'autre sexué, ne pouvait trouver d'autre issue qu'un refuge dans le giron de la bisexualité agie incarnée par la silhouette androgyne.

Elles effectuent ainsi un travail autour de l'acquisition d'une sexualité généralisée. Il s'agit là d'un changement qualitatif, avec une entrée dans l'univers de la signification. Elles sont dorénavant, par conséquent, davantage dans la représentation d'elles-mêmes que sous le sceau aliénant du signe, tels le donné à voir ou bien le corps donné en pâture. Pour elles, à présent, la sexualité peut s'auto-alimenter, signant leur accès à une autonomie en tant que sujet sexué. Leur discours indique qu'elles développent un imaginaire avec une différenciation sexuée.

Leur sexualité peut devenir généralisée avec une resexualisation du corps et de la pensée. Ces femmes accèdent à une dimension intégrale de l'humain dans ce questionnement vis-à-vis de la sexualité. D'après leur discours, il s'agit d'une tentative d'harmoniser leur division sur leurs différentes formes de sexualité agie en tant qu'épouses dépendantes du produit, concubines, prostituées, mères abandonnantes. En expérimentant l'accès à la substitution, 
elles nous disent percevoir que toutes les relations peuvent être vécues dans le respect des différences - le genre étant considéré par ces femmes comme un outil pour interroger les différentes structures sociales. Ainsi, leur accès au sexe social peut se donner à voir hors de la réalité effective de la différence des sexes. Cette différence est désormais appréhendée par le truchement de la relation à l'autre, effectuant le lien entre les deux sexes. Ces femmes se requalifient en qualité de personne par rapport à la relation à l'autre sexe. Maintenant, la relation prime sur l'un et l'autre sexe, dans un souci de construction sociale.

On peut considérer que ces femmes sortent de l'aliénation de la logique de la domination masculine où elles étaient transformées en objets ou en instruments. Ne pouvant exercer un pouvoir sur le cours de leur vie, elles étaient dominées par celui tout-puissant du produit censé résoudre les difficultés de la conflictualité psychique.

\section{L'engagement relationnel}

Par le biais de l'institution, en intégrant le corps social incarné par le soin au corps, l'abandon progressif des contraintes induites par le lien au produit entraîne une décorporéisation des actes. Cet exercice, maintes fois accompli sous forme de ritualisation sur l'autel de la médecine spécialisée en addictologie, amène les femmes subrepticement au cœur de la relation. Peu à peu, pour nommer la différence, ces mères utilisent en effet la relation. On assiste alors à leur propre recréation du rapport à soi, à l'enfant, à l'autre. Par ailleurs, cette relation qui s'instaure peut résulter d'un point de vue intégrant le changement à l'œuvre au cours de la prise en charge. On perçoit, en outre, que la narrativité de leur discours se pare de la mise en intrigue des relations entre le masculin et le féminin, thème central dans toute l'œuvre de LéviStrauss. De plus, elles font l'expérience que l'engagement relationnel repose également sur les notions de rareté et de valeur, d'où la question: "Qu'est-ce que je vaux ? » Ainsi, elles s'offrent la possibilité d'interroger le critère de la valeur à travers l'évocation des échanges sexuels et affectifs.

\section{Transmission et processus d'affiliation}

Nous percevons donc qu'au travers de leur discours ces femmes s'approprient un système de valeurs ; se soigner prend une signification par l'attribution d'un sens. Ainsi, elles se saisissent de cet ensemble de qualités véhiculé par l'institution médicale. On peut affirmer qu'ici c'est une des formes de la culture qui donne la clé de l'interprétation. Mais nous savons bien qu'elles ne sont pas encore sorties de l'institution, loin s'en faut, craignant peut-être d'être l'objet d'une infinie diversité de leurs perceptions 
individuelles si elles étaient autonomes hors du champ institutionnel de la substitution. On constate en effet que pour la plupart elles ne sont pas encore entrées dans le monde de l'échange symbolisé par la division du travail. En revanche, elles peuvent s'approprier le mythe individualiste par la singularité de l'acte d'ingérer la dose calibrée de méthadone. Enfin, pour entrer en relation avec l'enfant, ces mères en soin font exister les institutions. Pour C. Castoriadis (1999), celui qui prend en charge l'éducation doit s'autodestituer, la relation mère-bébé étant par nature deshistoricisée. Par la spécificité du soin apporté, ces mères sont prises chacune au sein d'un processus d'individuation : c'est ici la médecine, mandatée par la société, qui est chargée de les individuer. Leur discours laisse transparaître que par l'institution du langage elles ont accès à leur propre subjectivité qui, ellemême, est soumise à la mouvance.

Ainsi, le travail de ces femmes participe à la redéfinition globale du système de filiation délié des institutions autour du mariage, dans une dynamique de réaffiliation : il nous rappelle que se reproduire ne va pas de soi, n'est pas sans risque.

À l'inverse des régimes totalitaires qui livrent une sociologie politique de la construction de l'image du corps, une politique de soins dans un système démocratique s'emploie à réintroduire de l'histoire dans une construction culturelle de la différence des sexes pour une population qui s'est exclue de sa propre histoire.

\section{La narrativité comme auto-historisation}

Dans une perspective psychodynamique, chez les sujets rencontrés, la césure qui nous est rapportée est métaphorisée par l'introduction d'une vectorisation du temps : le temps du désir, être jeune fille, femme puis mère, mais aussi : avant la méthadone, après la contamination, etc., dans une adresse à l'autre qui en tisse une trame. Cet après-coup recréé permet au noyau traumatique du processus psychique (Janin, 2005) de se déployer sur au moins deux temps. Ce noyau traumatique représente, par exemple, le réel du pubertaire qui vient s'inscrire psychiquement à une période où l'excitation sexuelle a donné un sens rétrospectif au temps de la première inscription du traumatique. Nous avons à l'esprit la théorie freudienne préconisant que l'articulation de toute une série bien connue de couples d'opposés qui agissent au sein de toute vie mentale, produisant indéfiniment du traumatisme et du traumatique.

C. Janin (2005) nous parle de trois temps du traumatisme : le premier est celui qui n'est pas assimilé par le Moi ; le deuxième est un temps de sexualisation du premier temps traumatique; le troisième est post-pubertaire. Ce redéploiement des différents temps, par l'introduction de ces césures, fait effet de mise en sens : ce qui vient après dans la réalité psychique explique 
ce qui était survenu avant. Ainsi le symptôme, compromis auquel contribuent toutes les tendances issues du traumatisme, entre les ranimations du souvenir et son extinction, ne peut être compris que parce qu'il donne lieu à une construction de sa survenue. Alors une narrativité peut émerger, avec une mise en intrigue d'une histoire partageable qui se déplie au sein d'un engagement relationnel. Le récit de la trajectoire fait figure ici d'autohistorisation, fonction dévolue au moi dans une tentative d'autoreprésentation. Une succession d'après-coups peut alors donner sens à des événements qui, sans cela, seraient restés en suspens. Cette reprise élaborative va favoriser la remise en circulation de la force et de l'efficacité des représentations jusque-là figées dans des impressions traumatiques, selon F. Brette (2005). Nous rappelons ici que l'analyste, au cours de la cure, travaille à partir des après-coups que produit le transfert, quand se rejoue la névrose de transfert, par le biais des répétitions qui s'y trament, révélant la fixation au trauma. La situation analytique provoque inévitablement une resexualisation et l'excitation ainsi suscitée peut avoir une action traumatique, réactualisant un traumatisme infantile, selon F. Brette.

Par l'entremise des après-coups issus des interprétations offertes par le social, en prise avec la naissance de la parentalité, nous avons assisté là à un déplacement de l'acquisition d'une posture maternelle attendue vers celle, plus subreptice, d'une resexualisation du corps et de la pensée. Selon nous, les femmes rencontrées nous donnent à entendre une accession à la dimension intégrale de l'humain dans ce questionnement en tant que sujet sexué, dans l'évocation de la relation à elle-même, aux figures identificatoires, à l'homme, à l'enfant... Ainsi, se reproduire, loin de se limiter aux retrouvailles avec la mère des origines ou avec son propre infantile, est bien poser un acte porteur d'une nouvelle signification en se liant à un nouveau venu. On ne reproduit jamais du même. L'adulte, dans l'accession à sa parentalité, est en quête d'affiliation par la culture qui va lui donner la clé d'une interprétation recherchée. La réalisation des projets de vie serait alors le gage d'une accession à une identité pleinement investie, garantie d'une appropriation du parcours.

\section{Ouvertures}

Au cours de ce travail, nous avons tenté de déterminer en quoi l'accès à un traitement de substitution pour des mères toxicomanes peut s'inscrire dans la logique d'une trajectoire. Nous avons par ailleurs cherché à percevoir, à travers leur propre vision des choses, ce que l'usage de la méthadone engendre comme conséquences, non pas sur les processus de guérison des utilisatrices, mais sur leurs différents types de relation au monde : relation à elles-mêmes, aux ascendants et descendants, au conjoint en particulier. Un autre ensei- 
gnement de notre étude montre l'intérêt d'envisager une trajectoire dans sa totalité, plutôt que d'en isoler un seul élément.

Ainsi, nous pensons que les femmes, au cœur de notre étude, ont pu se réapproprier une image d'elles-mêmes plus valorisante par le récit de toutes les difficultés qu'elles ont traversées. Le traitement de substitution, s'inscrivant par nature dans la durée, autorise les usagers à effectuer un travail de recomposition des modes relationnels. La méthadone peut jouer un rôle de parrain, en soutenant une vectorisation du temps. Les femmes toxicomanes en traitement répartissent ainsi les événements constitutifs de leur vie en deux périodes distinctes pour lesquelles le temps n'a pas la même valeur: le temps du chaos d'avant la méthadone et le temps ordonnancé de la substitution.

Dans notre étude, les mères rencontrées disent être constamment en danger de désaffiliation au niveau tant de leur ascendance que de leur descendance. À partir de l'agencement sous la forme de récits qu'elles tentent de se réapproprier, ces femmes expriment leurs incertitudes quant à l'exercice de leur capacité à incarner les différentes facettes de leur féminité dans la relation à elles-mêmes, à l'autre et à l'enfant. L'étude de ce questionnement nous a permis une approche du féminin maternel qui serait la faculté de mise en forme, par le discours, des interrogations sur les différentes façons de s'engager dans l'interrelation.

Soulignons ici comment la survenue de la toxicomanie peut, en se reformulant dans le cadre d'un parcours, prendre alors sens dans l'histoire de chaque individu. Par le truchement du discours se déploient de nombreuses possibilités de mise en scène du féminin permettant aux femmes toxicomanes de traiter de manière créative les « images » fantasmagoriques de leur identité, comme des supports transitoires qui, dans leur diversité, jouent un rôle comparable aux représentations traditionnelles du féminin et, par-delà, autorisent l'émergence de la complexité de leur identité féminine. Ainsi, on peut laisser à ces femmes un jeu réel dans l'invention d'elles-mêmes, une liberté dans la disposition des images qu'elles voudront endosser ou refuser. La possibilité de narrer à un tiers leur relation à un autre être humain qui attire ou éveille un désir peut être considérée aussi comme une formation de soi-même dans une perspective érotique.

Dans notre étude, nous avons pris le parti de ne rencontrer que des mères sous substitution. En les interrogeant, il nous est rapidement apparu nécessaire de bénéficier de l'apport des récits des trajectoires des pères toxicomanes afin d'établir des correspondances éventuelles dans les modes d'accès à la relation à soi-même, à l'autre et à l'enfant. Un travail futur pourrait aborder l'étude de récits à deux voix dans une perspective qui prendrait en compte les aléas de la construction du parcours parental dans un souci de complémentarité. Ces recherches pourraient également explorer le rôle tiers de la substitution au sein de la dynamique d'un couple. Nous entrevoyons là un champ très ouvert pour de futures recherches, dans la mesure où la substitution est 
un processus de soin mis en place récemment par les pouvoirs publics. Un des enjeux de cette politique d'accompagnement, c'est d'offrir la possibilité à l'usager de déployer ses capacités afin de parvenir à réaliser ses projets de vie. Ces derniers seraient alors le gage d'une accession à une identité pleinement investie, garantie d'une appropriation du parcours. On assisterait à la transfiguration de la trajectoire qui se transmuterait en histoire, alors objet de transmission.

\author{
Ouriel Rosenblum \\ Service de psychiatrie de l'enfant et de l'adolescent, Salpêtrière, Paris \\ Laboratoire PSYTEC, Lille 3 \\ Laboratoire PSY-NCA, équipe TIF, Rouen \\ 15, clos Bruneau, 75005 Paris \\ rosenblouriel@noos.fr
}

\title{
Bibliographie
}

Angel, P. 2000. « La femme toxicomane », dans P. Angel, D. Richard et M. Valleur (sous la direction de), Toxicomanie, Paris, Masson.

BRETte, F. 2005. «Le traumatique: effets positifs et organisateurs », dans F. Brette, M. Emmanuelli et G. Pragier (sous la direction de), Le traumatisme psychique, Organisation et désorganisation, Paris, PuF, « Monographies de psychanalyse », 70.

Elias, N. 1991. La société des individus, Paris, Agora, Pocket.

JANIN, C. 2005. «Au cœur de la théorie psychanalytique : le traumatisme », dans F. Brette, M. Emmanuelli et G. Pragier (sous la direction de), Le traumatisme psychique, Organisation et désorganisation, Paris, PUF, « Monographies de psychanalyse », 43-55.

Losito, N. ; Gibier, L. 1999. «Couple et toxicomanie : dépendance mutuelle », Intervention, 70/71, 3-12.

Rosenblum, O. 2004. «Parentalité et toxicomanie », dans P. Angel et Ph. Mazet (sous la direction de), Guérir les souffrances familiales, Paris, PUF.

Steinberg, S. 2001. La confusion des sexes. Le travestissement de la Renaissance à la Révolution, Paris, Fayard.

THÉRY, I. 2001. « Mixité et maternité », dans Y. Knibiehler (sous la direction de), Maternité, affaire privée, affaire publique, Paris, Bayard, 251-170.

Tномаs, O. 2006. Toxicomanie féminine: du traumatisme sexuel à l'amour de transfert, Toulouse, érès.

\section{RÉSUMÉ}

Si les conduites addictives établissent une stratégie pour retarder l'entrée dans le champ de la sexualité adulte, la rencontre avec l'héroïne signe une satisfaction des besoins qui fait l'impasse sur le désir engagé par le corps sexué. Cet engagement dans l'addiction hypothèque la fertilité à travers les difficultés de l'investissement du corps chez les femmes toxicomanes. Une prise en charge spécifique, avec l'appui de produits de substitution, permet l'introduction d'une fonction de parrainage auprès des futurs parents.

\section{Mots-CLÉS}

Addiction, sexualité, désir d'enfant. 


\section{DRUG ADDICTION TOWARDS MOTHERLY FEMININITY}

SELF CENTERED PLEASURE TO SEXUALIZED RELATIONSHIP

\section{Summary}

While addictions lead to a strategy to delay access to adult sexuality, addiction to heroin satisfies certain needs that annihilate one's own sexual desire. This investment in addiction reduces fertility due to the difficulties addicted women have in caring for their bodies. A specific form of care with the help of drug substitutes can lead to the introduction of a sponsorship function for future parents.

\section{KEYwORDS}

Drug addiction, sexuality, broody feeling. 